\section{Carotenoids of a Red Toadstool, Phallus rugulosus}

\author{
Keiji Harashima \\ Department of Agricultural Chemistry, \\ The University of Tokyo, Bunkyo-ku, \\ Tokyo, Japan
}

Received June 2, 1978

Several species are known of Phallaceae forming red fruit bodies of penis- (or pencil-) like shapes. No investigation have been carried out on pigments contained in these toadstools. The author had chances of collecting fruit bodies of a toadstool, Phallus rugulosus (Fisch.) O. Kuntze, in the early summer of 1976 at Kinuta, Setagaya-ku in Tokyo, and analyzed their pigment composition. Two main pigments were obtained in crystalline states. This brief report concerns the identification of these pigments.

Frozen specimens of 13 fruit bodies weighing about $67 \mathrm{~g}$ were extracted several times with acetone until the residue became almost colorless. The combined extract was shaken with $n$-hexane and water, so that the pigments were transferred to the $n$-hexane layer, which was then washed with water repeatedly until acetonefree. When the $n$-hexane solution was then extracted 3 times with each $1 / 2$ volume of aqueous $95 \%(\mathrm{v} / \mathrm{v})$ methanol, more than $90 \%$ of the total pigments remained unextracted. The epiphasic layer was evaporated in vacuo, and the residue was dissolved in a minimum volume of warm $n$-hexane and cooled overnight at $-20^{\circ} \mathrm{C}$. Purple red crystals (crystal I) precipitated, which were recrystallized 3 times from warm $n$-hexane and then once from benzene-ethanol.

The mother liquor of crystal I was loaded on a column of aluminum oxide and the adsorbed pigments were eluted stepwise with $n$-hexane containing increasing concentrations $(1 \sim 10 \%)$ of acetone. The fraction eluting first (Fr. I) with $1 \%$ acetone was evaporated in vacuo and the residue was dissolved in a minimum volume of warm $n$-hexane and cooled overnight at $-20^{\circ} \mathrm{C}$. Red crystals (crystal II) obtained were recrystallized from warm $n$-hexane. Following Fr. I a fraction was eluted which formed an yellow band on the column and gave a visible absorption spectrum like that of neurosporene. The fractions adsorbed more strongly on the column separated not completely each other.

Crystal I as well as crystal II was completely epiphasic when it was distributed between $n$-hexane and aqueous $95 \%(\mathrm{v} / \mathrm{v})$ methanol. As illustrated in Fig. 1,

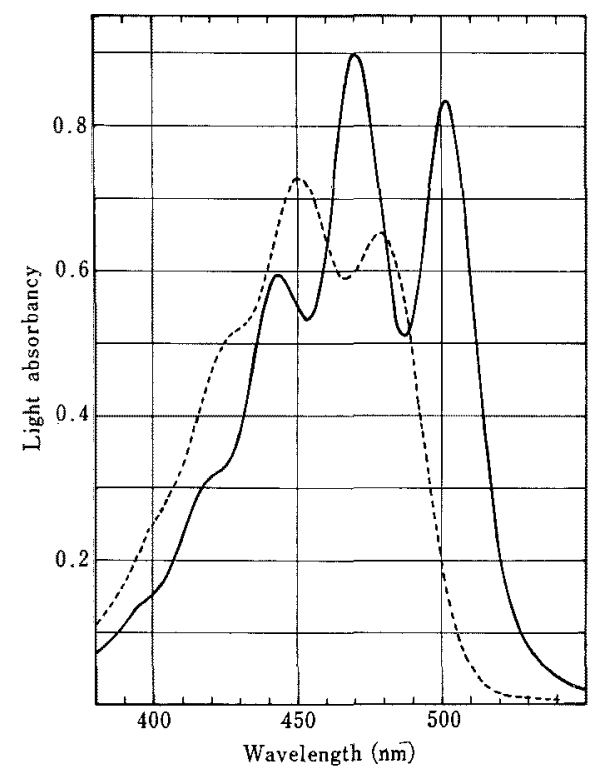

Fro. 1. Visible Absorption Spectra of Crystals I and II.

Crystals $I$ and II were dissolved in $n$-hexane in arbitraty concentrations and submitted to the recording of the absorption spectra with a Hitachi's spectrophotometer, Model 124. Solid line: crystal I, $\lambda_{\max }=$ $443 \sim 4,470 \sim 470.5$ and $501.5 \mathrm{~nm}$. Dotted line: crystal II, $\lambda_{\max }=450.5$ and $478.5 \mathrm{~nm}$.

crystals I and II showed visible absorption spectra identical to those of lycopene ${ }^{1 /}$ and $\beta$-carotene ${ }^{2)}$ respectively. On plates of magnesium oxide-Kieselguhr $G^{3)}$ crystals I and II did not separate from lycopene (isolated from tomato fruits) and synthetic $\beta$-carotene (purchased from F. Hoffman-La Roche \& Co. Ltd.) respectively. Mass spectrometric cracking patterns of crystals I and II (Fig. 2) were also identical to those of lycopene and $\beta$-carotene ${ }^{4)}$ respectively. These results show that crystal I is identical with lycopene and crystal II with $\beta$-carotene.

Analysis of mother liquor of crystal II ( $\beta$-carotene) by TLC on Magnesium oxide-Kieselguhr $\mathrm{G}^{3>}$ showed that Fr. I did not contain any carotenoid other than a set of stereoisomers of $\beta$-carotene. Total amount of epiphasic carotenoids was calculated to be about $1.9 \mathrm{mg}$ (as lycopene), of which about $0.8 \mathrm{mg}$ was recovered in crystal $I$ (lycopene). About $0.3 \mathrm{mg}$ of $\beta$ carotene was found in the mother liquor of crystal I.

Acknowledgement. The author withes to express cordial thanks to Dr. Yoshimichi Doi, National Science Museum, for his kind and helpful advice for the identification of Phallus rugulosus. 

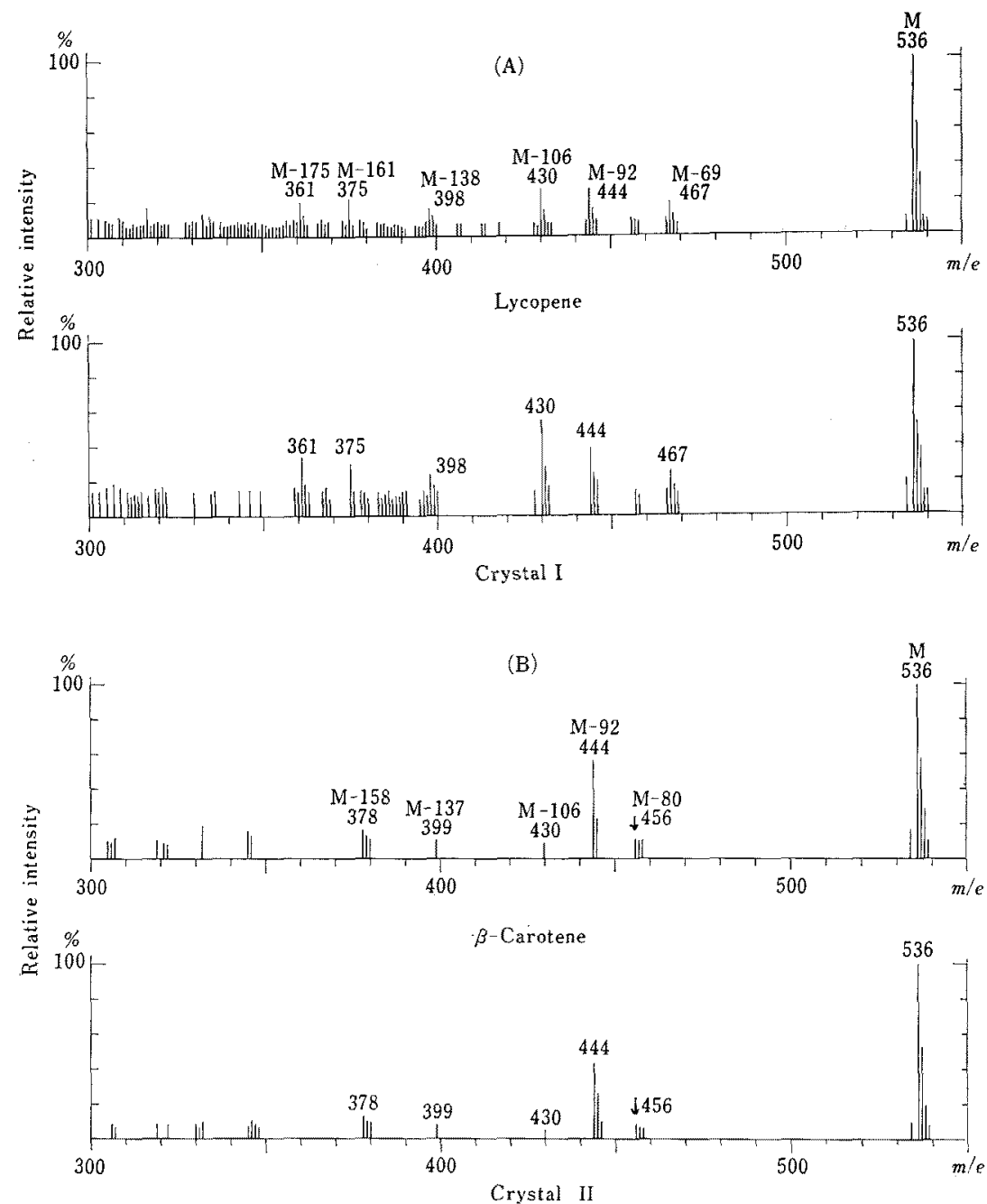

FIG. 2. Mass Spectra of Crystals I and II, Obtained on a Hitachi's Mass Spectrometer RMU-6 $\left(170 \sim 200^{\circ} \mathrm{C}, 70 \mathrm{eV}\right)$.

Relative intensities of fragment peaks were represented by fractions of molecular peaks.

A: mass spectra of crystal I and lycopene.

B: mass spectra of crystal II and $\beta$-carotene.

\section{REFERENCES}

1) L. Zechmeister, A. L. Le Rosen, W. A. Schroeder, A. Polgár and L. Pauling, J. Am. Chem. Soc., 65, 1940 (1943).
2) L. Zechmeister and A. Polgár, J. Am. Chem. Soc., 65, 1522 (1943).

3) K. Harashima, T. Ohno, T. Sawachika, T. Hidaka and E. Ohnishi, Insect Biochem., 2, 29 (1972).

4) U. Schweiter, G. Englert, N. Rigassi and W. Vetter, Pure Appl. Chem., 20, 365 (1969). 